\title{
Delayed elective surgery in a major teaching hospital in Uganda
}

\author{
Isaac Kajja' \\ Cees Th Smit Sibinga ${ }^{2}$ \\ 'Department of Orthopedics, \\ Makerere University, Kampala, \\ Uganda; ${ }^{2}$ ID Consulting for \\ International Development of \\ Transfusion Medicine (IDTM), \\ Groningen, The Netherlands
}

Correspondence: Isaac Kajja Department of Orthopedics, Makerere University, PO Box 7072, Kampala, Uganda

Tel +256 772443473

Emailkajjal33@gmail.com
This article was published in the following Dove Press journal:

International Journal of Clinical Transfusion Medicine

7 May 2014

Number of times this article has been viewed

Background: A number of factors come into play in determining the timing of an elective surgical intervention, particularly in the developing world. The present study explores the factors that contribute to the timing of elective surgery and patients' opinions on their quality of life as they wait for surgery.

Methods: We followed adult patients with delayed elective surgical interventions $(n=204)$. The causes for the delay and, particularly, the influence of blood shortage on the timing of the procedure were noted. Patients' perceptions on their quality of life as they waited for surgery were also noted.

Results: We were able to establish a cause for delayed elective surgery in 133 patients. Shortage of operating space was the leading cause of surgery delay in 44 patients, while blood shortage followed closely in 40 patients. The higher the amount of blood ordered for use in the perioperative time, the longer the delay to surgery $(P=0.001)$. Patients waiting for surgery had a low opinion of their in-hospital quality of life. Here, the key indicators included the threat of losing a job, limited family time, and an increase in day-to-day living costs.

Conclusion: Blood shortage is the second most common cause of the delayed performance of elective surgical interventions in our institution. The patients have a low opinion on their quality of life as they wait for surgery.

Keywords: blood shortage, delayed elective surgery, quality of life

\section{Introduction}

An elective surgical operation provides time for the attending health team (surgeon, anesthesiologist, nursing, laboratory, and operating room staff) to perform the necessary clinical and laboratory workup before an appropriate date and time are set for the procedure. The emotional and psychological effects following admission for elective surgery can be accentuated by delay of performance of the intended surgical intervention. ${ }^{1,2}$ In developing countries, the delay in the performance of these elective procedures is largely due to bottlenecks in the existing health system essentials. These include lack of adequate infrastructure, like operating theater space, admission beds, diagnostic equipment, and intensive care units. Shortage of a dedicated, well-trained, and motivated human resource (technical and support) cadre is another cause for these delays. Additionally, inconsistent supply of recurrent utilities like medication and theater supplies hinders timely performance of elective surgical procedures. ${ }^{3-6}$ Delayed elective surgery may lead to increased morbidity and mortality for the patient, but it may also result in high hospital costs given the prolonged hospital stay. ${ }^{7}$ 
The elective interventions come as a result of a set indication and as part of a clinical treatment plan, while the emergency situation needs immediate intervention. It has been established that in the Department of Orthopedics at Mulago Hospital (Kampala, Uganda), patients presenting with a fractured femur shaft - which should have been handled with an immediate surgical intervention - take a minimum of 5 days' admission on a hospital bed before they undergo their intended surgery. ${ }^{8}$ Thus, patients requiring urgent surgery are admitted to the hospital, receive initial resuscitation, but their procedures are not performed as expected and scheduled. Patients are kept waiting for their definitive surgical procedure and end up being documented as elective cases. It is hypothesized that many of the elective surgical interventions in this hospital should have been handled as emergency cases, had there been no bottlenecks in the institutional infrastructure and other health systems. It is further hypothesized that one of the major causes for delayed execution of scheduled surgeries includes a shortage of blood supply for use in the perioperative time.

The aims of this study were, first, to review the causes of delay in the execution of elective surgeries. Secondly, we explored whether or not blood shortage could be one of the causes for delayed elective surgery at Mulago Hospital. Thirdly, this study served as a pilot study to assess the health and socioeconomic implications of delayed surgery, for which we analyzed the perceptions of patients regarding how delayed surgery affects their in-hospital quality of life.

\section{Methods}

At Mulago Hospital, Uganda's national referral and major teaching hospital of 1,500 beds, patients are admitted and undergo the appropriate clinical assessment, as well as a laboratory and radiological diagnostic workup, after which a decision to undertake a surgical intervention is made. Pretransfusion blood samples are drawn for grouping and crossmatching of the required blood for those patients for whom the attending surgeon anticipates the use of blood before, during, or after surgery. Within a distance of $2 \mathrm{~km}$ is a regional blood bank - Nakasero Blood Bank (Kampala, Uganda) which supplies blood to the Mulago Hospital after performing the standard screening procedure for transfusion transmitted infections on each unit collected. In this hospital, there is no maximal surgical blood order schedule followed in any of the departments performing elective surgical procedures. Second, the national blood policy does not allow any cost recovery from the patient or from a health facility for the supplied blood. So, the availability of blood for an elective surgery is determined on a first-come, first-served basis, and is supply driven. Third, up to $50 \%$ of the blood supplied by Uganda Blood Transfusion Services (UBTS; Kampala, Uganda) to hospitals is used for hemotherapy in the management of severe childhood anemia due to malaria. While $30 \%$ is used for the management of emergency obstetric hemorrhage, only $20 \%$ remains for the adult medical, emergency trauma, and elective surgical procedures. This is made worse by the lack of a well-defined and analyzed need for blood, for example, for elective surgery in Uganda. ${ }^{9}$

The Mulago Hospital has a total of 23 operating rooms distributed, as shown in Table 1, among the various surgical disciplines. It has a total of eight anesthesiologists and 33 anesthetists managing the entire surgical load for the hospital. In Uganda, an anesthesiologist is an individual who is trained up to a master's degree level, while an anesthetist is trained up to a diploma level in anesthesia.

This cross-sectional audit was conducted at Mulago Hospital between October 1, 2012 and November 30, 2012 in the departments of Orthopedics, Obstetrics and Gynecology (Obs and Gyn), and General Surgery (administratively, all surgical disciplines shown in Table 1, other than Orthopedics and Obs and Gyn, are under General Surgery). This urban teaching and referral hospital handles elective surgical procedures that are referred from all over the country, and from neighboring South Sudan, the Democratic Republic of Congo, Rwanda, Kenya, and Tanzania. The hospital was commissioned more than 50 years ago and has seen minimal expansions despite an increasing catchment area with its accompanying growing population and increasingly high disease burden.

Table I The available operating space per surgical discipline

\begin{tabular}{ll}
\hline Surgical discipline & $\begin{array}{l}\text { Number of operating } \\
\text { rooms }\end{array}$ \\
\hline Emergency surgery & 2 \\
Accident and emergency & 2 \\
Emergency obstetrics & \\
Elective surgery & 3 \\
Orthopedics & $\mathrm{I}$ \\
Spine & $\mathrm{I}$ \\
Burns & $\mathrm{I}$ \\
Cardiac surgery & 3 \\
Gynecology & 2 \\
Ophthalmology & 2 \\
Ear, nose, and throat & $\mathrm{I}$ \\
Maxillofacial & $\mathrm{I}$ \\
Urology & $\mathrm{I}$ \\
Breast and endocrine surgery & $\mathrm{I}$ \\
Hepatobiliary surgery & $\mathrm{I}$ \\
Colorectal & $\mathrm{I}$ \\
Neurosurgery & 23 \\
Total &
\end{tabular}


Following clearance from the hospital institutional review board, 854 consecutive adult patients ( 18 years and above who had been scheduled for elective surgical interventions in the departments mentioned earlier) were recruited after seeking verbal consent from each of the patients to participate in the study. Patients who had comorbidities other than the diagnosis for which the surgery had been planned were excluded. In this study, a delay to surgery was defined as any surgery not performed for 1 day or more from the day the surgery had been planned. Perioperative time was taken as 48 hours before to 48 hours after the surgical intervention.

A pretested questionnaire was used to collect data. The demographic variables included: sex; age; preoperative hemoglobin level; and clinical diagnosis. The rank of the surgeon, as well as the surgeon's planning and taking responsibility for the entire surgical procedure were documented. The surgical diagnoses were categorized as major if the surgeon anticipated that the patient's perioperative blood loss would be $500 \mathrm{mLs}$ or more, and minor if the surgeon anticipated the patient to lose less than $500 \mathrm{mLs}$ of blood in the perioperative time. The amount of whole blood (in units of $450 \mathrm{~mL}$, as supplied by UBTS) ordered for perioperative use was noted. The date on which the surgery had been planned to take place, and the actual date on which the surgery was performed, as well as the cause of delay to perform a surgical procedure, as reported by the attending surgeon, were noted. Additionally, the patients' perceptions of their in-hospital quality of life during the waiting time to come to surgery were recorded. If the patient perceived the clinical condition for which the surgical procedure had been planned to have worsened during the waiting time, it was noted as "disease deterioration". Any patient who could not be allowed by his employers to resume his routine duties due to prolonged hospital stay was noted as "lost a job". If a patient developed any other clinical condition during his or her hospital stay, it was noted as "developed another disease". Report of dissatisfaction from family members due to a patient's absence from the family was noted as "lost family time".

For analytical purposes, the duration of the delay was categorized as $0-1$ week, $1-2$ weeks, and more than 2 weeks. Data were analyzed using the SPSS statistical software package (version 12.0; IBM Corporation, Armonk, NY, USA). Data were reported as proportions, mean \pm standard deviation and range. The chi-square test was performed to compare categorical variables, and the independent $t$-test was used to compare differences in the means of continuous variables. A nonparametric test (Kruskal-Wallis) was done to ascertain the relationships between multiple categorical variables. Statistical significance was taken at $P<0.05$.

\section{Results}

During the study period, 854 patients underwent elective surgery, 204 (23.9\%) of whom had their surgeries delayed (Table 2). Up to $75.2 \%$ of all elective surgery performed in the Department of Orthopedics was delayed compared to $20.9 \%$ and $10.95 \%$ of delayed elective surgical interventions performed in the Obs and Gyn and General Surgery departments, respectively. The male-to-female ratio among the 204 patients was 1:1 (males: number $[\mathrm{n}]=102$; females: $\mathrm{n}=102$ ) with a mean age of $39.9 \pm 20.0$ years. Preoperative hemoglobin levels ranged from $4.0-15.6 \mathrm{~g} / \mathrm{dL}$ with a mean of $10.4 \pm 2.7 \mathrm{~g} / \mathrm{dL}$. The surgical diagnosis for $178(87.2 \%)$ patients was categorized as major, while $26(12.8 \%)$ patients had their diagnoses categorized as minor. Out of the 178 major categorized diagnoses (anticipated loss of $500 \mathrm{~mL}$ or more in the perioperative time), 86 were from the Department of Orthopedics, 54 from the Obs and Gyn department, and 38 from the General Surgery department. Most of these 204 patients $(n=88$ [43.1\%]) were under the responsibility of a consultant surgeon, while medical officers cared for only seven (3.4\%) patients (Table 3 ).

A cause for the delay in surgical intervention could be established in $133(65.2 \%)$ patients, while we were not able to find the reason for this delay in $71(34.8 \%)$ patients. The delay was more than 2 weeks for 75 out of the 133 patients. Standalone causes for the delay to surgery included a lack of theater space in 44 (33.1\%) cases, a lack of blood to use in perioperative time in 40 (30.1\%) patients, and poor working condition of the anesthesia equipment in two $(0.02 \%)$ patients. It was identified that in $47(35.3 \%)$ patients, the delay to surgery was due to more than one cause. These included: shortage of an anesthesiologist or anesthetist; delayed consent to surgery; hemodynamic instability; and lack of recurrent theater supplies like oxygen (Table 4).

Table 2 The surgical procedures performed during the study period

\begin{tabular}{llll}
\hline Department & $\begin{array}{l}\text { Emergency } \\
\text { surgeries }\end{array}$ & $\begin{array}{l}\text { Elective } \\
\text { surgeries }\end{array}$ & $\begin{array}{l}\text { Delayed } \\
\mathbf{N}(\%)\end{array}$ \\
\hline $\begin{array}{l}\text { Orthopedics } \\
\text { Obstetrics and }\end{array}$ & 217 & 129 & $97(75.2 \%)$ \\
gynecology & 1,546 & 304 & $61(20.1 \%)$ \\
General surgery & $25 \mathrm{I}$ & $42 \mathrm{I}$ & $46(10.9 \%)$ \\
Total & 2,014 & 854 & $204(23.9 \%)$ \\
\hline
\end{tabular}

Note: $\mathrm{N}(\%)=\mathrm{N}$ of delayed surgeries as a percentage of the elective surgeries performed in a given department.

Abbreviation: $\mathrm{N}$, number. 
Table 3 Demographics of the studied patients

\begin{tabular}{ll}
\hline Variable & Characteristic \\
\hline Age & Mean: $39.9 \pm 20.0$ years (range: I8-87 years) \\
Sex & Male $=102 ;$ females $=102$ \\
Hemoglobin* & Mean: $10.4 \pm 2.7 \mathrm{~g} / \mathrm{dL}$ (range: $4.0-15.6 \mathrm{~g} / \mathrm{dL}$ ) \\
Category of procedure & Major $=178 ;$ not major $=26$ \\
Surgeon cadre & MO $=7 ; \mathrm{SHO}=60 ;$ Reg $=13 ;$ Con $=88 ;$ \\
(patient number)** $^{*}$ & $\mathrm{~S} /$ Con $=36$
\end{tabular}

Notes: $*$ Hemoglobin = preoperative hemoglobin; **surgeon cadre (patient number) = number of patients whose surgical procedures were under a particular rank of surgeons. Results are represented as mean \pm standard deviation.

Abbreviations: $\mathrm{MO}$, medical officer; $\mathrm{SHO}$, senior house officer; Reg, registrar; Con, consultant surgeon; S/Con, senior consultant surgeon.

A total of 143 units of whole blood ( $450 \mathrm{~mL}$ each) were ordered for 70 patients with a mean of 2.04 \pm 1.08 units per patient. Out of these 70 patients, $61(87.1 \%)$ had their scheduled surgeries delayed; 40 were solely due to blood shortages, and 21 were due to a blood shortage and another cause. The attending surgeon tended to delay the surgical intervention for a longer period of time, when a higher amount of blood to transfuse in the perioperative time was anticipated for a given patient ( $P=0.001)$ (Table 5).

Forty-seven patients whose surgery had been delayed responded to the question of perceived in-hospital quality of life during the waiting time to surgery (Table 6). Disease deterioration was reported by 25 patients, and there were a total of eight lost jobs and eight individuals who lost family time, while development of another disease during hospital stay and high day-to-day costs were reported by three patients, respectively. Differentials in the effects on the quality of life, as perceived by these patients, were not statistically significant $(P=0.07)$.

\section{Discussion}

Within the 2 months of the study period, the three surgical departments handled more than 2,000 surgical procedures. Notable among the study population the hemoglobin level

Table 4 The extent of the delay to surgery in relation to the cause of the delay

\begin{tabular}{llllll}
\hline $\begin{array}{l}\text { Delay } \\
\text { in weeks }\end{array}$ & $\begin{array}{l}\text { Lack of } \\
\text { theater } \\
\text { space }\end{array}$ & $\begin{array}{l}\text { Lack of } \\
\text { blood }\end{array}$ & $\begin{array}{l}\text { Lack of } \\
\text { anesthesia }\end{array}$ & Others & Totals \\
\hline $0-1$ & 6 & 9 & 0 & 8 & 23 \\
I-2 & II & 6 & $\mathrm{I}$ & 17 & 35 \\
$>2$ & 27 & 25 & $\mathrm{I}$ & 22 & 75 \\
Total & $44(33.1 \%)$ & $40(30.1 \%)$ & $2(0.02 \%)$ & $47(35.3 \%)$ & 133 \\
\hline
\end{tabular}

Notes: Delays to surgery caused by two or more of the following: lack of theater space; lack of blood; lack of anesthesia; shortage of an anesthesiologist or anesthetist; delayed consent to surgery; hemodynamic instability; and lack of recurrent theater supplies, like oxygen. Lack of anesthesia = total of equipment, anesthesiologists and anesthetists.
Table 5 The extent of the delay to surgery in relation to the amount of blood ordered

\begin{tabular}{llllll}
\hline Delay in & \multicolumn{4}{l}{ Number of units of whole blood ordered } \\
\cline { 2 - 6 } weeks & $\mathbf{I}$ unit & $\mathbf{2}$ units & $\mathbf{3}$ units & $\mathbf{4}$ units & $\mathbf{6}$ units \\
\hline $0-\mathrm{I}$ & 9 patients & $\mathbf{8}$ patients & 0 patients & 0 patients & 0 patients \\
I-2 & 7 patients & 3 patients & I patient & 0 patients & 0 patients \\
$>2$ & 4 patients & I 4 patients & 9 patients & 4 patients & 2 patients \\
Total & 20 & 25 & 10 & 4 & 2 \\
patients & & & & & \\
\hline
\end{tabular}

Note: The data represent the number of patients for whom a particular amount of blood was ordered.

was relatively low compared to the same age category in the general population. The most common causes of low hemoglobin levels in Uganda's adult population are poorly managed intestinal helminthiasis, malnutrition, and anemia due to chronic diseases like human immunodeficiency virus/ acquired immunodeficiency syndrome. This large patient load comes with compromised in-hospital health care and poor patient outcomes. The senior surgeons were available to make decisions and plan for the elective surgeries, but these could not be performed within the planned time due to institutional factors like a shortage of operation theater space. In a comparable tertiary-care teaching public-sector hospital in the developing Caribbean island, Barbados, Jonnalagadda et al $^{10}$ identified a number of administrative and infrastructural hindrances to the timely performance of elective surgical procedures. The majority of cancellations of elective surgical interventions were due to a shortage of beds, nurses, and recurrent supplies like linen. Chalya et al, ${ }^{11}$ in a comparable setting, identified delays in surgical interventions in $81.4 \%$ of their series of 3,064 patients, which were due to a lack of operation theater space and facilities.

This study has further revealed that the availability of blood for use during the perioperative time is a key determinant for the timing of elective surgery. Bathla et $\mathrm{al}^{12}$ have identified blood shortages as one of the causes of delayed elective surgery in India. At Mulago Hospital, an elective surgical procedure will be delayed if the attending surgeon makes an order for blood to be used in the perioperative time. Additionally, the more blood that is ordered, the longer the patient has to wait for surgery. In Uganda, the blood needs and the clinical dynamics pertaining to the in-hospital transfusion practices are not fully appreciated by the supplying blood establishment. ${ }^{9}$ Likewise, the clinicians do not fully comprehend the challenges faced by UBTS in the procurement, processing, and distribution of blood to hospitals. This lack of a sound clinical interface leads to the observed erratic supply and subsequent shortage of blood for use in surgery. ${ }^{13}$ Second, the lack of a structured and functional hospital transfusion 
Table 6 The effects of delayed surgery, as reported by patients, and as related to the cause of the delay

\begin{tabular}{|c|c|c|c|c|c|c|}
\hline Cause of the delay & $\begin{array}{l}\text { Disease } \\
\text { deteriorated }\end{array}$ & $\begin{array}{l}\text { Developed } \\
\text { another disease }\end{array}$ & $\begin{array}{l}\text { Lost family } \\
\text { time }\end{array}$ & Lost job & $\begin{array}{l}\text { High day- } \\
\text { to-day costs }\end{array}$ & Total \\
\hline Blood shortage & 18 patients & I patient & 5 patients & 2 patients & I patient & 27 (57.4\%) \\
\hline Lack of theater space & 3 patients & 0 patients & 2 patients & 3 patients & 2 patients & 10 \\
\hline \multirow[t]{2}{*}{ Others } & 4 patients & 2 patients & I patient & 3 patients & 0 patients & 10 \\
\hline & 25 & 3 & 8 & 8 & 3 & 47 \\
\hline
\end{tabular}

committee leads to the inappropriate clinical use of blood exemplified by unguided blood ordering culture in surgical practice. ${ }^{8}$ In this study, 20 out of the 61 patients whose surgery was delayed had a request for only one unit of blood. This would have been offset by the use of other blood-saving approaches like oral ferrous sulfate to build the patients' hemoglobin before surgery. We postulate that out of desperation, the attending surgeon had to order at least a unit of blood for use in the perioperative time, given the anticipated blood losses in the major surgeries, the prevalent low preoperative hemoglobin levels in the patient population, and the perennial short supply of blood for use in surgery in Uganda. ${ }^{13}$ In such situations, surgery had to be postponed until at least a unit of blood had been supplied to the operating room for a given patient, hence the observed delays. Additionally, the patient load to this hospital overstretches the blood supply, leading to the denial of blood to some patients in preference to others, especially for hemotherapy in childhood anemias and in obstetric hemorrhage. In an editorial, Lipton et al ${ }^{14}$ have earmarked the importance of the supply of adequate and safe blood for all medical and surgical disciplines in the medium and low human development index countries: the UNDP use a human development index (HDI) classification of countries with four classifications - low, medium, high and very high. This has not been fully appreciated by the development partners of these countries and has led to the inadequate supply of blood, particularly for the surgical disciplines; hence, the observed delays to elective surgery in our institution.

Patient opinions on the health care services provided by a given health facility are paramount in ensuring quality improvement and customer satisfaction. It is the basis for building customer confidence in the health care system..$^{15,16}$ As a self-audit tool, this study has revealed that patients have a low opinion of the quality of in-hospital life while waiting for surgery, as shown by 25 out of 47 respondents who reported deterioration in their diagnosed disease. Although only 47 patients responded to the questionnaire, the delay to surgery could have affected the post-hospital quality of life of most of the patients who were included in this study. Additionally, the three patients who reported high day-to-day costs while in hospital could represent a tip of the iceberg of the vast expenses incurred by patients in this cosmopolitan national teaching and referral hospital as they wait for surgery. In a related study, Petrie et al ${ }^{17}$ studied a series of 100 patients in Canada who identified that economic hardships were a key psychological and social economic concern among patients waiting for coronary artery bypass surgery.

In summary, this study has established that a number of factors come into play to delay a planned surgical procedure. The availability of an operating space is the most prevalent cause of these delays. It is further revealed that the availability of blood for use in the perioperative time can be a key determinant for the timing of elective surgery. In a setting without clear hospital policies on blood use in surgery, a step-by-step quality improvement strategy at the clinical interface should be established. This should start with the implementation of a well-supported and functioning hospital transfusion committee to oversee all blood transfusion activities. This will also guide the definition of blood need in surgical practice, as well as the development and implementation of maximal surgical blood order schedules for the various performed surgical procedures, and it will lead to improved blood inventory management in the hospital setting. Additionally, delayed elective surgery negatively impacts the patient, the hospital, and the community at large. Therefore, we advise that all concerned stakeholders should advocate for spending resources on a detailed study of the socioeconomic implications of delayed surgery and come up with timely and acceptable remedial strategies.

\section{Disclosure}

The authors report no conflicts of interest in this work.

\section{References}

1. Al-Ghazal SK, Sully L, Fallowfield L, Blamey RW. The psychological impact of immediate rather than delayed breast reconstruction. Eur $J$ Surg Oncol. 2000;26(1):17-19.

2. Fernández-Delgado J, López-Pedraza MJ, Blasco JA, et al. Satisfaction with and psychological impact of immediate and deferred breast reconstruction. Ann Oncol. 2008;19(8):1430-1434.

3. Kierans C, Padilla-Altamira C, Garcia-Garcia G, Ibarra-Hernandez M, Mercado FJ. When health systems are barriers to health care: challenges faced by uninsured Mexican kidney patients. PLoS One. 2013;8(1): e54380. 
4. Hsia RY, Mbembati NA, Macfarlane S, Kruk ME. Access to emergency and surgical care in sub-Saharan Africa: the infrastructure gap. Health Policy Plan. 2012;27(3):234-244.

5. Adamu A, Maigatari M, Lawal K, Iliyasu M. Waiting time for emergency abdominal surgery in Zaria, Nigeria. Afr Health Sci. 2010;10(1): 46-53.

6. Cadotte DW, Viswanathan A, Cadotte A, Bernstein M, Munie T, Freidberg SR; East African Neurosurgical Research Collaboration. The consequence of delayed neurosurgical care at Tikur Anbessa Hospital, Addis Ababa, Ethiopia. World Neurosurg. 2010;73(4):270-275.

7. Zoucas E, Lydrup ML. Hospital costs associated with surgical morbidity after elective colorectal procedures: a retrospective observational cohort study in 530 patients. Patient Safety in Surgery. 2014;8:2.

8. Kajja I, Bimenya GS, Eindhoven B, Jan Ten Duis H, Sibinga CT. Blood loss and contributing factors in femoral fracture surgery. Afr Health Sci. 2010;10(1):18-25.

9. Kajja I. The current hospital transfusion practices and procedures in Uganda. Academic Thesis University of Groningen, Groningen, NL. 2010. ISBN: 978-90-367-4619-9.

10. Jonnalagadda R, Walrond ER, Hariharan S, Walrond M, Prasad C. Evaluation of the reasons for cancellations and delays of surgical procedures in a developing country. Int J Clin Pract. 2005;59(6):716-720.

11. Chalya PL, Gilyoma JM, Mabula JB, et al. Incidence, causes and pattern of cancellation of elective surgical operations in a university teaching hospital in the Lake Zone, Tanzania. Afr Health Sci. 2011;11(3): $438-443$.
12. Bathla S, Mohta A, Gupta A, Kamal G. Cancellation of elective cases in pediatric surgery: an audit. J Indian Assoc Pediatr Surg. 2010;15(3): 90-92.

13. Kajja I, Bimenya G, Smit Sibinga C. The interface between blood preparation and use in Uganda. Vox Sang. 2010;98(3 Pt 1):e257-e262.

14. Lipton KS, Reilly J, Ness PM. Poverty and human development: access to a safe and adequate supply of blood for transfusion. Transfusion. 2007;47(11):1952-1954.

15. Tait AR, Voepel-Lewis T, Munro HM, Gutstein HB, Reynolds PI. Cancellation of pediatric outpatient surgery: economic and emotional implications for patients and their families. J Clin Anesth. 1997;9(3):213-219.

16. Dadaş S, Eti-aslan F. The causes and consequences of cancellations in planned orthopaedic surgery: the reactions of patients and their families. Journal of Orthopaedic Nursing. 2004;8(1):11-19.

17. Petrie JF, Cox JL, Teskey RJ, Campbell LB, Johnstone DE. Preliminary assessment of patients' opinions of queuing for coronary bypass graft surgery at one Canadian centre. Qual Health Care. 1996;5(3): $166-171$.
International Journal of Clinical Transfusion Medicine

\section{Publish your work in this journal}

International Journal of Clinical Transfusion Medicine is an international, peer-reviewed, open access, online journal publishing clinicalexperimental, policy-making and evidence-based practices of all topics pertaining to clinical transfusion medicine. Original research, short reports, reviews, case reports and commentaries are invited.

\section{Dovepress}

The manuscript management system is completely online and includes a very quick and fair peer-review system, which is all easy to use. Visit http://www.dovepress.com/testimonials.php to read real quotes from published authors. 